\title{
Nonlinear Dynamics of Ionization Fronts in HII Regions
}

A. Mizuta, J. O. Kane, M. W. Pound, B. A. Remington, D. D. Ryutov, H. Takabe

May 7, 2006

HEDLA '06 Conference

Houston, TX, United States

March 11, 2006 through March 14, 2006 
This document was prepared as an account of work sponsored by an agency of the United States Government. Neither the United States Government nor the University of California nor any of their employees, makes any warranty, express or implied, or assumes any legal liability or responsibility for the accuracy, completeness, or usefulness of any information, apparatus, product, or process disclosed, or represents that its use would not infringe privately owned rights. Reference herein to any specific commercial product, process, or service by trade name, trademark, manufacturer, or otherwise, does not necessarily constitute or imply its endorsement, recommendation, or favoring by the United States Government or the University of California. The views and opinions of authors expressed herein do not necessarily state or reflect those of the United States Government or the University of California, and shall not be used for advertising or product endorsement purposes. 


\title{
Nonlinear Dynamics of Ionization Fronts in HII Regions
}

\author{
Akira Mizuta ${ }^{1}$, Jave O. Kane ${ }^{2}$, Marc W. Pound ${ }^{3}$, Bruce A. Remington ${ }^{2}$, Dmitri D. Ryutov ${ }^{2}$, and Hideaki \\ Takabe $^{4}$
}

\begin{abstract}
Hydrodynamic instability of an accelerating ionization front (IF) is investigated with 2D hydrodynamic simulations, including absorption of incident photoionizing photons, recombination in the HII region, and radiative molecular cooling. When the amplitude of the perturbation is large enough, nonlinear dynamics of the IF triggered by the separation of the IF from the cloud surface is observed. This causes the second harmonic of the imposed perturbation to appear on the cloud surfaces, whereas the perturbation in density of ablated gas in the HII region remains largely single mode. This mismatch of modes between the IF and the density perturbation in the HII region prevents the strong stabilization effect seen in the linear regime. Large growth of the perturbation caused by Rayleigh-Taylor-like instability is observed late in time.
\end{abstract}

\section{Introduction}

Columns or pillars are common structures seen in HII regions. One of the most popular examples of this structure is the Eagle Nebula, which has three famous pillars beside some O stars (Hester et al. (1996)). The boundary between the cloud and HII region is an ionization front (IF) where photoevapolation occurs, resulting in photoevapolated flow. The HII region consists of almost fully ionized hydrogen and is isothermal at $T \sim 10^{4}$ $\mathrm{K}$. The inside of the pillars consists of dense and cold molecular hydrogen. The hydrogen number density in the pillar in the Eagle Nebula is $\sim 10^{4}-10^{5} \mathrm{~cm}^{-3}$ and the temperature is about a few tens of Kelvins (Pound 1998). Pound found the velocity gradient along pillar from the head to the bottom (see also Pound et al. 2006, Kane et al. 2006 in this volume). Dense clumps, some of which will become young stars, are observed in the pillar (McCaughrean \& Andersen(2002)). Since this cloud is optically thick for incident photons, the thickness of the IF is very thin. Because of the similarity of this phenomena with laser ablation, scaled laboratory experiments using laser ablation are proposed to study IF dynamics (Kane et al. 2005).

It is still not fully understood how the pillars form, though several hypotheses have been proposed. For example, some models are based on hydrodynamic instability of the IF. Spitzer (1954) proposed a model that the shape of pillars are at due to nonlinear phase of the Rayleigh-Taylor instability which occurs when a light fluid accelerates a denser fluid. Vandervoort (1962) theoretically found unstable modes at an IF without acceleration, but the important role recombination plays in the HII region was not included. Axford (1964) extended Vandervoort's work, including recombination and found that recombination in the HII region works to stabilize the perturbation, as suggested by Kahn (1958). Sysoev (1997) did more complete analysis and found the growth of long-wavelength instabilities for normally incident radiation. Williams (2002) confirmed this with 2D simulations. He also included effects of the radiation tilt in the analysis. The effect of tilted rays of the incident radiation to the IF with acceleration was theoretically studied by Ryutov et al. (2003,2006). Recently Mizuta et al. (2005) have numerically studied an accelerating IF and concluded that stabilization due to recombination works even with acceleration in the linear regime (i.e. small amplitude of the perturbations). This paper discusses cases with larger initial imposed perturbation.

\footnotetext{
${ }^{1}$ Max-Planck-Institute für Astrophysik, Karl-Schwarzschild-Str. 1, 85741 Garching, Germany E-mail mizuta@MPA-Garching.MPG.DE

${ }^{2}$ University of California, Lawrence Livermore National Laboratory, 7000 East Ave., Livermore, CA 94551 U.S.A.

${ }^{3}$ Department of Astronomy, University of Maryland, College Park, MD 20742 U.S.A.

${ }^{4}$ Institute of Laser Engineering, Osaka University, 2-6 Yamada Oka, Suita, Osaka, 565-0871, Japan
} 


\section{Model}

We studied the dynamics of an accelerating IF by varying the initial amplitude of the imposed perturbations. The 2D hydrodynamic equations are solved using the code described in Mizuta et al. (2005). The energy equation includes sources of cooling and heating due to absorption of the incident photons, recombination in the HII region, and radiative molecular cooling. The transport equation for the incident photons is also solved, considering the photon absorption by neutral hydrogen and recombination of ionized hydrogen. Recombination to the ground state is ignored, assuming that the diffusive photon is locally absorbed (on the spot approximation). See Mizuta et al. (2005) for more details.

A $0.46 \mathrm{pc} \times 3 \mathrm{pc}$ computational box with $184 \times 1200$ uniform grid points is used. Periodic boundary conditions are employed at $x=0$ and $x=0.46 \mathrm{pc}$. Outflow boundary condition are imposed at $y=0$ and $y=3$. A quarter pc thickness finite cloud is located at a distance of $0.5 \mathrm{pc}$ from the boundary at $y=3 \mathrm{pc}$, where the incident photon flux comes in. The hydorgen number density $n(H)$ of the cloud and other region are $10^{5}$ and $10 \mathrm{~cm}^{-3}$, respectively. The region $y<2.5 \mathrm{pc}$ is isothermal with $T=40 \mathrm{~K}$ and the region $y>2 \mathrm{pc}$ is pressur matched. A constant incident photon number flux of $5 \times 10^{11} \mathrm{~cm}^{-2} \mathrm{~s}^{-1}$ is taken to be parallel to the $y$ axis. When a neutral hydrogen atom absorbs an incident photon and becomes ionized, an internal energy increment of $1.73 \times 10^{-12} \mathrm{erg}$ is locally deposited into the gas. An isothermal state whose temperature is about $10^{4}$ Kelvin is achieved in the HII region as a result of the balance between this heating by photon absorption and cooling by the recombination of the ionized hydrogen. The molecular cloud is very cold ( $\sim 40$ Kelvin) due to the strong radiative molecular cooling, even if shocks cross in the cloud.

A sinusoidal surface perturbation is initially imposed. Here we have studied four different initial amplitude perturbations: $3.8 \times 10^{-3}$ pc (case S038), $9.0 \times 10^{-3}$ pc (case S090), $1.4 \times 10^{-2}$ pc (case S140), and $1.9 \times 10^{-2}$ pc (case S190), respectively. Figure 1(a) shows the number density contour of the initial condition, where the initial amplitude is $9.0 \times 10^{-3} \mathrm{pc}$.

\section{Results and Discussions}

Figure 2 shows the amplitude of the perturbation as a function of time, where the amplitude is defined as half the peak-to-valley amplitude of the contour corresponding to an ionization fraction of $f=0.5$. Since the IF sometimes separates from the cloud surface, this amplitude shown in Fig 2 does not always correspond to the amplitude of the perterbation at the cloud surface. Case S038 shows a small amplitude which oscillates with time, and does not grow, as shown in Mizuta et al. (2005). When the incident photon flux comes in, the flow of the photoionized plasma begins from cloud surface. Since this flow is normal to the cloud surface, the density of the photoevaporated flow around the bubble region (surface concavities at $x=0,0.46 \mathrm{pc}$ ) becomes higher than around spike region $(x=0.23 \mathrm{pc})$, due to focusing effect. Higher number density results in stronger absorption of the incident photon flux in the HII region. As a result, the ablation pressure around the bubble region becomes lower than that around the spike region. The local difference of the ablation pressure works to strongly stabilize the perturbation in linear regime.

The amplitude of the other cases, however, increase, with time, in striking contrast to the strong stabilization observed in the linear regime. The reason is due to the "separation of the IF". The strong absorption of incident photon around the bubble occurs, reducing the ablation pressure there, also in the larger initial amplitude case. The surface perturbation inverts phase completely during the shock propagates in the dense cloud (Fig. 1 (b)(c)). The number density around the bubble region becomes higher as the IF becomes strongly concave. The increasing density around the bubble in the HII region causes absorption of all incident photons before the cloud surface as shown in Fig. 1 (d), since the number of incident photons is finite. This causes the separation of the 
IF from the cloud surface, which has not been considered before. When the separation of the IF from the cloud surface occurs, a tiny and warm HI region (neutral hydrogen) appears between the cloud surface and the IF, since the gas was once ionized and recombined to neutral hydrogen. The cloud surface locally does not feel any ablation pressure and expands in the $y$ direction, when the separation of the IF appears.

After a few tens of kyr, the IF corresponds to the cloud surface again. But the perturbation on the cloud surface is not longer single mode; a second harmonic of the imposed perturbation has appeared (Fig. 1 (e)). To the contrary the density perturbations in the HII regions above the cloud surface are still single mode (Fig. 1 (e)). This mismatch between the perturbation modes on the cloud surface and those in the density of the ablated plasma mean that the stabilization observed in the linear regime will not occur. (Recall, this stablization requires that these two perturbations be "mode locked" 180 degrees out of phase.) As a result, a Rayleigh-Taylor-like instability evolves and large growth of the second harmonic of the imposed perturbation appears (Fig. 1 (f)-(h)).

We can measure the velocity gradient along the $y$ axis as Pound observed in the pillars in the Eagle Nebula. We find the velocity gradient to be about $12 \mathrm{~km} \mathrm{~s}^{-1} \mathrm{pc}^{-1}$ in the central column at $t=480 \mathrm{kyr}$. This is good agreement with observed one (an average magnitude of $8.3 \mathrm{~km} \mathrm{~s}^{-1} \mathrm{pc}^{-1}$ ) ${ }^{5}$.

The dynamics described in this paper is good target to model the scaled laboratory experiments, since theoretical analysis is difficult to do because of the nonlinear dynamics. We will demonstrate some numerical simulations to seek the possible laboratory experiments to study the instability presented in this paper.

\section{Conclusion}

We present a new type of the instability for the accelerating IF triggered by the separation of the IF from the cloud surface. When the initial amplitude of the perturbation is small enough, the strong stabilization is observed. When the surface becomes concave, the number density increases. Strong absorption through this region locally reduces the ablation pressure as theoretically discussed by Axford (1964) for the non-accelerating IF.

To the contrary, when the initial amplitude of the perturbation exceeds a critical value, roughly when the ratio of the initial amplitude to wavelength is greater than 0.02 , the nonlinear dynamics is triggered by the separation of the IF. The separation of the IF causes the appearance of the second harmonic of the imposed perturbation. The perturbation in density in the HII region, however, remains largely single mode. This mismatched of the modes between the perturbations on the cloud surface and in the density of the ablated flow prevents the stabilization effect seen in the linear regime. A kind of Rayleigh-Taylor instability takes over. The large growth of the second harmonic of the imposed perturbation is observed in the later phase.

Acknowledgments Work performed under the auspices of the U.S. Department of Energy by the Lawrence Livermore National Laboratory under Contract No. W-7405-ENG-48 and with support from NASA Grant NRA 00-01-ATP-059 and from National Science Foundation under Grant No. AST-0228974. MWP supported by NSF Grant No. AST-0228974.

\section{References}

[] Axford, W. I. 1964, ApJ, 140, 112

[] Hester, J. J., et al. 1996, AJ, 111, 2349

\footnotetext{
${ }^{5}$ this value does not include the effect of the inclination angle
}

This work was performed under the auspices of the U. S. Department of Energy by University of California, Lawrence Livermore National Laboratory under contract W-7405-Eng-48. 
[] Kane, J. O. et al., 2005, Ap\&SS, 298, 261

[] Kane, J. O. et al., 2006, Ap\&SS, this issue

[] Kahn, F. D. 1958, Rev. Mod. Phys., 30, 1058

[] McCaughrean, M. J., \& Andersen, M. 2002, A\&A, 389, 513

[] Mizuta, A. et al. 2005, ApJ, 621, 803

[] Mizuta, A. et al. 2006, submitted to ApJ

[] Pound, M. W. 1998, ApJ, 493, L113

[] Pound, M. W. et al. 2006, Ap\&SS, this volume

[] Sysoev, N. E. 1997, Astronomy Letters, 23, 409

[] Ryutov, D. D. et al., 2003, Plasma Physics and Controlled Fusion, 45, 769

[] Ryutov, D. D. et al., 2006, 2006, Ap\&SS, this volume

[] Spitzer, L. J. 1954, ApJ, 120, 1

[] Vandervoort, P. O. 1962, ApJ, 135, 212

[] Williams, R. J. R. 2002, MNRAS, 331, 693 

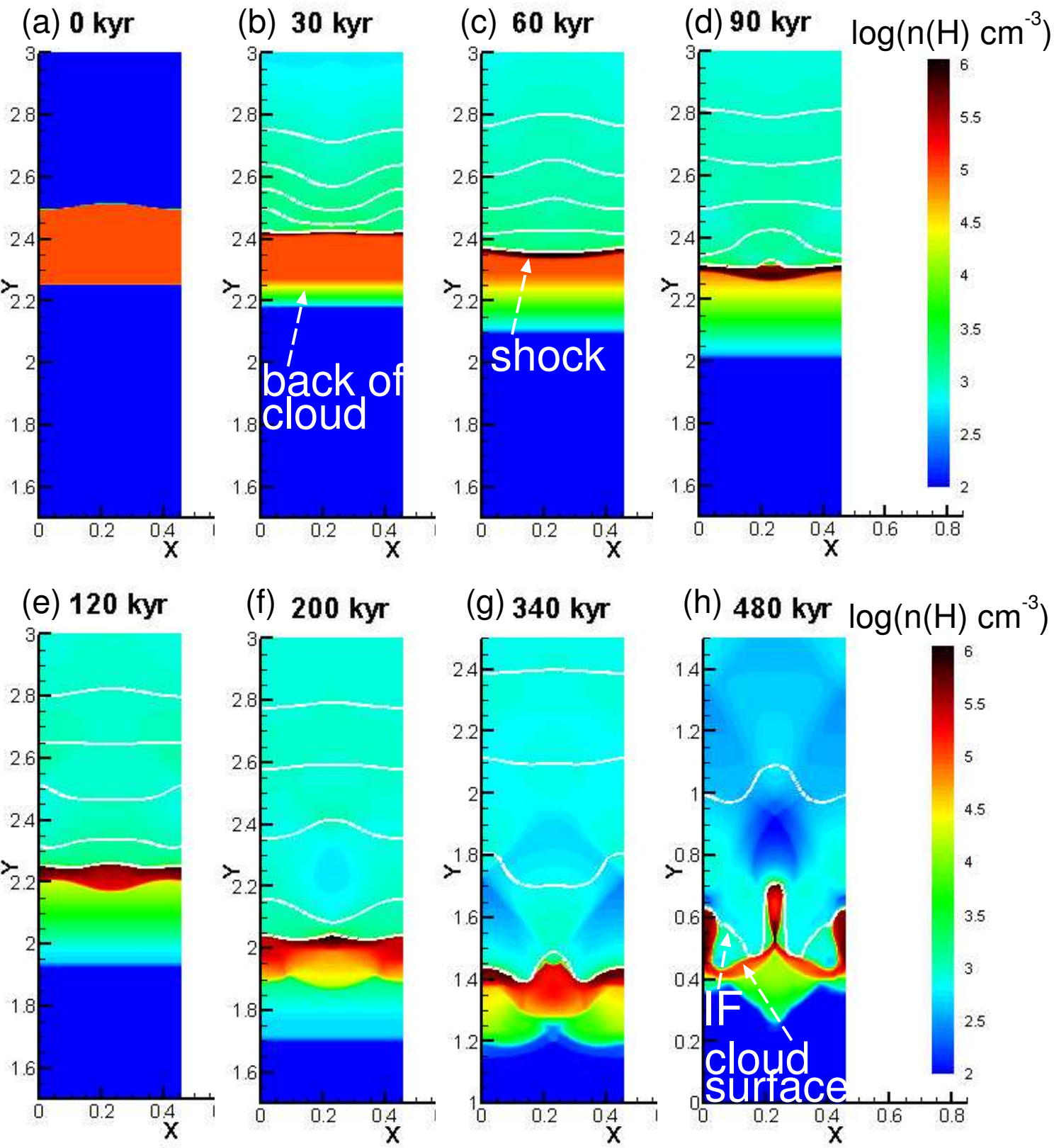

Figure 1: Number density contours (color) and incident photon number flux contours (solid white) at intervals of $1 \times 10^{11} \mathrm{~cm}^{-2} \mathrm{~s}^{-1}$, starting from the IF where ionization fraction $(f)$ goes to zero. The times shown correspond to (a) 0kyr, (b) $30 \mathrm{kyr}$, (c) $60 \mathrm{kyr}$, (d) $90 \mathrm{kyr}$, (e) $120 \mathrm{kyr}$, (f) $200 \mathrm{kyr}$, (g) $340 \mathrm{kyr}$ and (h) $480 \mathrm{kyr}$, respectively. As an example, the IF is indicated with an arrow in (f), a case where there is clear separation with the cloud surface (ablation front). The separation of the IF can be seen in (d), resulting the appearance of the tiny and warm HI region (neutral hydorgen atom). 


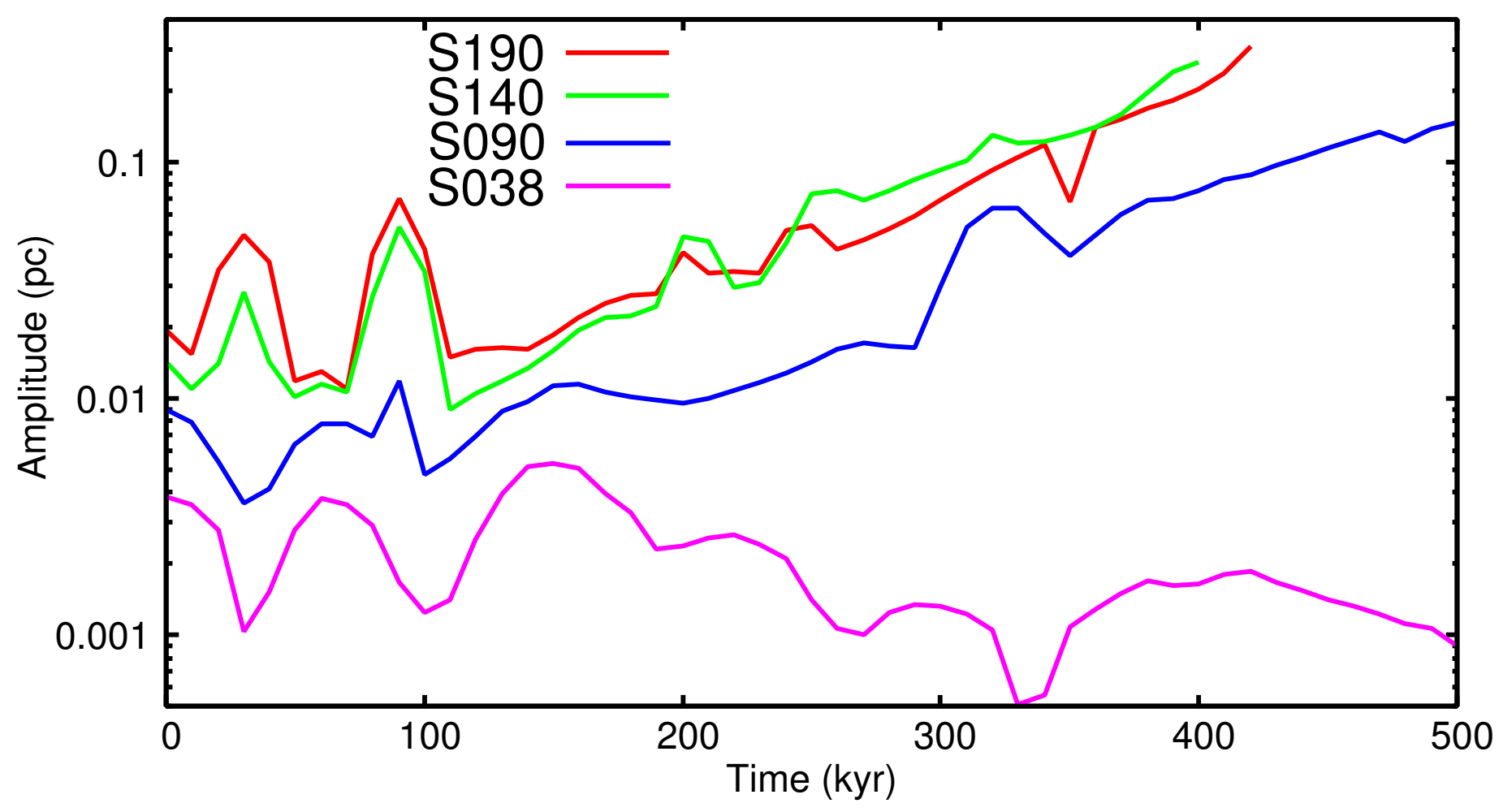

Figure 2: Time evolution of the "amplitude" of the perturbation for cases S038, S090, S140, and S190. 